\title{
Aerosol Therapy in Tracheotomized Children: Time for Guidelines!
}

There is an increasing number of ventilated patients who require aerosol medications, for various indications. While there are many studies of aerosol delivery through tracheostomy and endotracheal tubes in patients on mechanical ventilation, and also in patients with noninvasive positive pressure ventilation, ${ }^{1}$ a relatively neglected area of aerosol therapy is in non-ventilated patients, and particularly in infants and children, with tracheostomy.

\section{See the Original Study on Page 1234}

Tracheostomies in children are usually indicated when long-term cardiorespiratory compromise is anticipated or when a fixed airway obstruction is present and is unlikely to resolve for a protracted period. ${ }^{2}$ With regard to aerosol delivery in tracheotomized children, there is relatively little literature, and guidelines or consensus statements are lacking. In an American Thoracic Society 2000 consensus statement, aerosol administration via tracheostomy to children was clearly discouraged:

\begin{abstract}
Because the distribution, deposition, systemic absorption, local toxic effects, and efficacy of other (nonapproved for aerosol use) medications given via aerosol through a tracheostomy are unknown, the committee recommends avoiding such administration unless appropriate studies are performed. Drugs that are safely nebulized in mouth-breathing patients may achieve toxic concentrations in situations other than those originally studied. ${ }^{3}$
\end{abstract}

This month's ReSPIRATORY CARE contains a very interesting and comprehensive review by Willis and Berlinski about these issues. ${ }^{4}$ The authors have recently investigated aerosol delivery in a pediatric tracheostomy model. ${ }^{5}$ They have reviewed the existing literature and surveyed the respiratory care departments in numerous major United States institutions with pediatric pulmonology training programs regarding the technique of aerosol delivery to spontane-

The authors have disclosed no conflicts of interest.

Correspondence: Israel Amirav MD, Pediatric Department, Ziv Medical Center, Bar-Ilan University, Safed, Israel. E-mail: amirav@012.net.il.

DOI: $10.4187 /$ respcare.02005 ously breathing tracheotomized children. The survey revealed a variety of approaches, including some that had previously been shown to decrease drug delivery efficiency.

Surprisingly, despite widespread use of aerosol administration in tracheotomized children, there are no consensus statements or guidelines that incorporate the research published in the past decade. Why has no such consensus been achieved? Could it be because pharmaceutical companies have little commercial interest in supporting such efforts, or because official expert groups do not think there are enough patients who might benefit from such guidelines? The present study refutes this latter view!

Since many hospitals provide aerosol therapy to tracheotomized children, it is surely time that pediatricians, respiratory therapists, aerosol scientists, otolaryngologists, and pulmonologists convene a focus group to establish evidence-based guidelines for what are currently rather uninformed and often less than optimal approaches to therapy. It would be appropriate for respiratory therapists to take the lead in arranging such a consensus conference.

Israel Amirav MD

Pediatric Department

Ziv Medical Center

Bar-Ilan University

Safed, Israel

Michael T Newhouse MD MSc FRCP(C)

Barnett Medical Aerosol Research Laboratory

McMaster University

Hamilton, Ontario, Canada

\section{REFERENCES}

1. Dhand R. Aerosol therapy in patients receiving noninvasive positive pressure ventilation. J Aerosol Med Pulm Drug Deliv 2012;25(2): 63-78.

2. Trachsel D, Hammer J. Indications for tracheostomy in children. Paediatr Respir Rev 2006;7(3):162-168.

3. American Thoracic Society. Care of the child with a chronic tracheostomy. This official statement of the ATS was adopted by the ATS Board of Directors July 1999. Am J Respir Crit Care Med 2000; 161(1):297-308.

4. Willis LD, Berlinski A. Survey of aerosol delivery techniques to spontaneously breathing tracheostomized children. Respir Care 2012; 57(8):1234-1241.

5. Berlinski A, Hayden J. Albuterol delivery via nebulizer in a spontaneously breathing pediatric tracheostomy model. J Aerosol Med Pulm Drug Deliv 2011;24(3):57. 